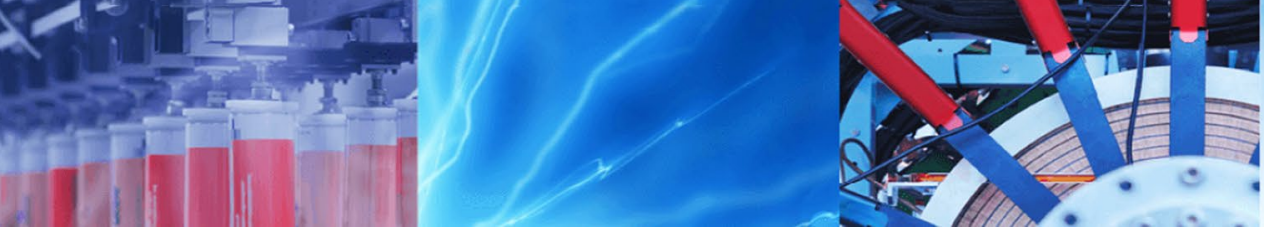

Research Article

\title{
Human postural ankle torque control model during standing posture with a series elastic muscle-tendon actuator
}

\author{
Mohammad Javad Fotuhi ${ }^{1}$. Orkun Yılmaz ${ }^{1} \cdot$ Zafer Bingul $^{1}$
}

Received: 2 November 2019 / Accepted: 3 January 2020 / Published online: 18 January 2020

(c) Springer Nature Switzerland AG 2020

\begin{abstract}
The main motivation behind the creation of a compliant actuation system is to provide safety, capability of storing energy, and improved performance levels in dynamic behavior of the mechanical human ankle. In this paper, a torsional flat spring (TFS) is proposed to provide high compliance and deformation values for human ankle series elastic muscletendon actuator system (HA-SEMTAS). The proposed torsional flat spring uses two torsional flat spiral spring in opposite directions, as the torsional flat spiral spring generates torque only in one direction. First, we present the characteristics of the TFS for an ankle joint, and the design and modeling of the HA-SEMTAS are developed. Modelling of the HA-SEMTAS simulates the responses of fluctuations in the center of mass and center of pressure and offers the possibility of measuring sensor activation, decomposition of reactive torque and participation of each set of muscle groups to balance posture. In order to control ankle angles and torque of Human system, torque controllers (PID and PD-feedforward) are generated for (HA-SEMTAS). The controller inputs are torsional flat spring position errors and error rates. Feedforward and integral action were applied to reduce the steady-state error of the system. It is seen that PID + ff of the system developed here is robust to step and ramp-type disturbances. Real-time controllers are embedded in GoogolTech GT-800 Industrial PC. Experimental results confirm that the step tracking of human upright posture behavior is satisfactory and overall system stability has improved using the proposed controller and compliant actuation system. It is also shown that PD + feedforward yields better performance than PID controller.
\end{abstract}

Keywords Series elastic actuator · Postural control · Ankle strategy · Pd-feedforward · PID

\section{Introduction}

Human stability related diseases are becoming more common with the increase in the average age of the people $[1,2]$. With ageing, risk of falling in daily life inevitably increases. These falls can cause various injuries like cracks and fractures in elderly people. This type of injury reduces the quality life. Furthermore, even if these injuries can be treated, some complications after surgeries can be dangerous.

Studies about postural stability are vital to gain more understanding about self-balance mechanisms of the human body. Postural stability can be defined as the ability to adjust the displacement of the body's centre of pressure (CoP) to react to the movement of the centre of gravity (CoG). Internal and external disturbances that more dynamic than body's reaction time can cause to fall. In order to work on postural control, various systems were created in previous studies. After studying the literature, two basic and different perspectives can be found for bipedal posture control. One of them is biologically inspired (BI) approach which can be called sensory-based approach. Humans process the data collected from vestibular system, muscle spindles (proprioceptive) and force

Mohammad Javad Fotuhi, mohammad.fotuhi@kocaeli.edu.tr; Orkun Yılmaz, orkun.yilmaz@kocaeli.edu.tr; Zafer Bingul, zaferb@kocaeli.edu.tr | ${ }^{1}$ Robotics and Automation Laboratory, Mechatronics Engineering Department, Kocaeli University, Kocaeli, Turkey. 
sensors to control their balance. $\mathrm{BI}$ approach has been developed based on this knowledge and a multi-sensory system is used for estimation of CoG's position and orientation values and compensation of disturbances in order to control balance $[3,4]$. The other widely used viewpoint is the robotics approach which is a model-based method. Unlike the $\mathrm{BI}$ in this approach, a dynamic model of the body is required. In [5-7], model-based bipedal control systems were developed.

Morphological reconstructions are commonly used for decreasing the complexity of the dynamic model without distorting biomechanical behaviors. Since ankle joint performs the most effective task of maintaining equilibrium in sagittal plane, a simplified single inverted pendulum model (SIP) can be substituted over the exact model in lower extremity bipedal balance problems [8].

In this study, we designed and built a lower extremity system to work on the postural stability control in the sagittal plane. The main difference of the proposed system is the series elastic actuator we added to ankle joint that named as human ankle series elastic muscletendon actuator (HA-SEMTAS). Since elastic series actuators are similar to skeletal muscles' structure, their use in the system resemble the response more like human [9]. HA-SEMTAS consists of a torque motor with two encoders (one of them is directly connected to the motor shaft and the other one is on the output shaft), a worm gear, a spring. Another contribution of our study is the design of Torsional flat spring. In order to change the stiffness of HA-SEMTAS, various types (thickness, materials) of these springs are manufactured.

The rest of our article was created as follows. In Sect. 2, the mathematical model of HA-SEMTAS is presented. Section 3 presents the control engineering framework, describes its main features and discusses how they are compared to those of the biological analysis. In Sect. 4 postural controller design is shown. Finally, Sect. 5 gives the main conclusions.

\section{Material and methods}

This section describes the details about mechanical design, mathematical model and proposed controller design of the presented HA-SEMTAS.

\subsection{HA-SEMTAS mechanical design}

Mechanical design of the HA-SEMTAS. The actuator components include the following.

- Frameless torque motor

- Worm gear

SN Applied Sciences

A SPRINGER NATURE journal
- Torsional flat spring

- Incremental encoders

- Link and joint of the ankle

The torque motor model is LFTM-50, which has a highresolution encoder. The rotary motion of the torque motor is converted to the other axis motion by a worm gear with the use of the bullring. The well of worm gear mechanism is connected to the link of ankle case and transmits the motion to the carriage where torsional flat spring is placed between them. The transmission ratio of the motor and worm gear system is 1:60. The spring constant is $63.665 \mathrm{Nm} / \mathrm{rad}$ and has a working stroke of $1 \mathrm{rad}$. The designed system is shown in Fig. 1.

For the adaptation of the elastic tendon actuator (Fig. 1), it is assumed that the elastic elements integrated in the tendons can be approximated as massless springs. This assumption is valid if the springs' mass is small compared to the effective mass of gearbox inertia and the motor and the effective mass of the joint and attachment. In the case of motors, this is especially true when using high reduction ratios gearboxes. The effective mass of the link is normally also higher by at least one magnitude. Figure 1 shows an overview of important parameters and variables of the series elastic muscle-tendon actuator.

\subsection{Torsional flat spring}

The proposed torsional flat spring (TFS) is composed of two preloaded spiral springs in opposite directions (see Fig. 2). To apply torque on the shaft through the springs, the worm of gearbox is rotated by a motor which is directly connected to the worm wheel. The torsional flat spring is rotated by a worm wheel, then is directly connected to the ankle joint and rotates the ankle link [10]. Also, an encoder is installed to measure the angle of joint. The difference between the angle of the motor and the angle of the joint is the torsion angle of the torsional flat spring.

The spring design procedure begins with the specifications about the TFS stiffness and maximum torque.

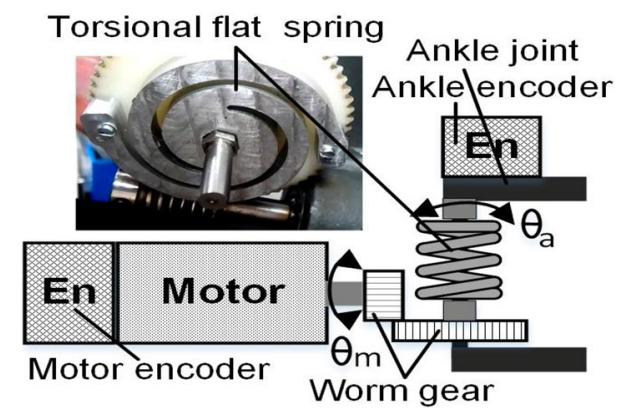

Fig. 1 Series Elastic muscle-tendon actuator system 


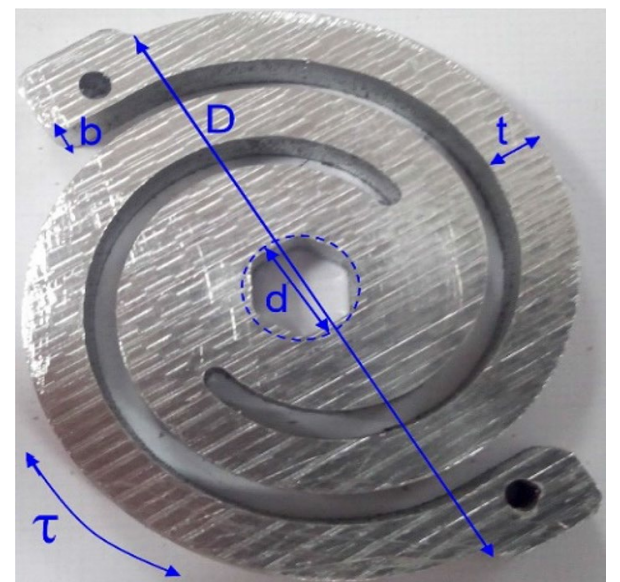

Fig. 2 Configuration and parameters of torsional flat Spring. The outside spring diameter, inside spring diameter, spring width, spring thickness, and torque on spring are denoted by $D, d, b, t$, and, $\tau$, respectively.

Since the TFS has two preloaded springs in opposite directions, the stiffness of one spring is one half of the required stiffness [11]. The maximum torque of one torsional flat spring can be determined once the preloading angle is chosen, which is explained in the following section. Once the specifications on the stiffness and maximum torque of the TFS are determined (see Fig. 3), the parameters can be obtained using (Eq. 1) given the material. Since there are more parameters to be determined than the number of equations, the dimension can be selected in terms of (2) and (3) to satisfy the size specifications.

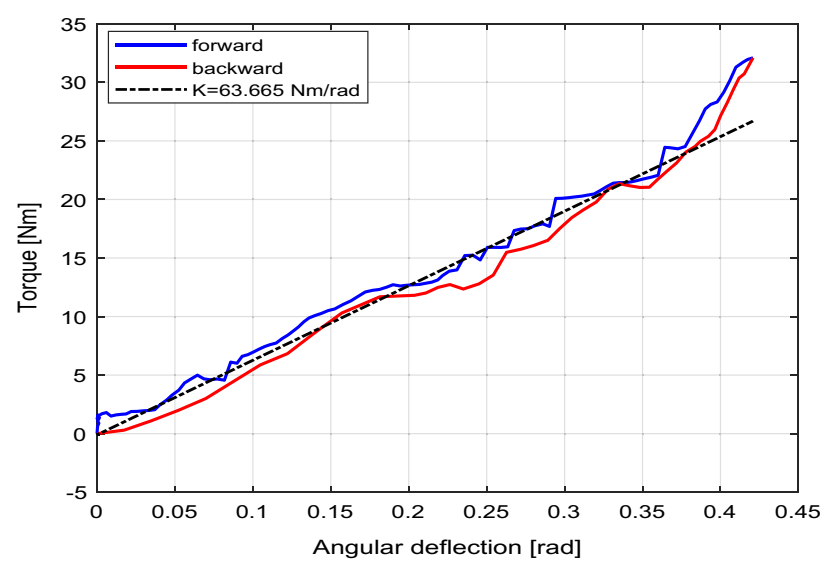

Fig. 3 Torque angle characteristic of the spring depicted on Fig. 2. The $93 \%$ interval confidence is given by the gray area around the curves but can barely be noticed (the standard deviation for different curves is less than $0.17 \mathrm{Nm}$ )
$k=\frac{E b t^{3}}{12 L}, \quad S=\frac{6 \tau}{b t^{2}}$

The torsional flat spring in HA-SEMTAS has been selected such that the TFS has a similar level of maximum torque with the ankle in the standing and has approximately $10 \%$ of the stiffness of the ankle.

$L_{\max }=\frac{D^{2}-d^{2}}{2.55 * t}$

$15<\frac{d}{t}<25, \quad \frac{L}{t}>1000$

The TFS dimensions and the system parameters are given in Table 1 . System has $80 \%$ of the maximum torque of the ankle [12].

\subsection{Modeling of HA-SEMTAS}

In the research on human postural stabilization, the stiff rod inverted pendulum model consisting of point mass located at the end of the rod is often used. This model represents sufficiently human posture during slow walking and during balancing in the presence of small disturbances (Fig. 4). Postural correction is achieved by the torque produced in the ankle joint (the pivot point). Denotingby $\tau_{\text {pivot }}$ the torque in this joint and by $\tau_{\text {in }}$ the torque due to inertial and gravity forces we can write the torque balance equation $\tau_{\text {pivot }}-\tau_{\text {in }}=i \ddot{\theta}_{a}$. Then the torque $\tau_{\text {pivot }}$ is

$\tau_{\text {pivot }}=\tau_{a}=1 \ddot{\theta}_{a}+\tau_{\text {in }}$

where $\tau_{\text {in }}=-x_{c} F_{z}+z_{c} F_{x}$ and:

$F_{x}=m \ddot{x}_{c}=-m l \sin \left(\theta_{a}\right) \dot{\theta}_{a}^{2}+m l \cos \left(\theta_{a}\right) \ddot{\theta}_{a}$

$$
\begin{aligned}
F_{z} & =m \ddot{z}_{c}+m g \\
& =-m l \cos \left(\theta_{a}\right) \dot{\theta}_{a}^{2}+m l \sin \left(\theta_{a}\right) \ddot{\theta}_{a}+m g
\end{aligned}
$$

Table 1 Selected TFS parameters

\begin{tabular}{lll}
\hline Parameter & Symbol & Mean value \\
\hline Outside diameter & $D$ & $61.41 \mathrm{~mm}$ \\
Inside diameter & $d$ & $11.97 \mathrm{~mm}$ \\
Width & $b$ & $7.3 \mathrm{~mm}$ \\
Thickness & $t$ & $4.87 \mathrm{~mm}$ \\
Length & $L$ & $112.73 \mathrm{~mm}$ \\
Stiffness & $k_{s}$ & $63.655 \mathrm{Nm} / \mathrm{rad}$ \\
Max deformation & $\Delta \theta$ & $0.4 \mathrm{rad}$ \\
Max torque & $\tau_{\max }$ & $25 \mathrm{Nm}$ \\
\hline
\end{tabular}



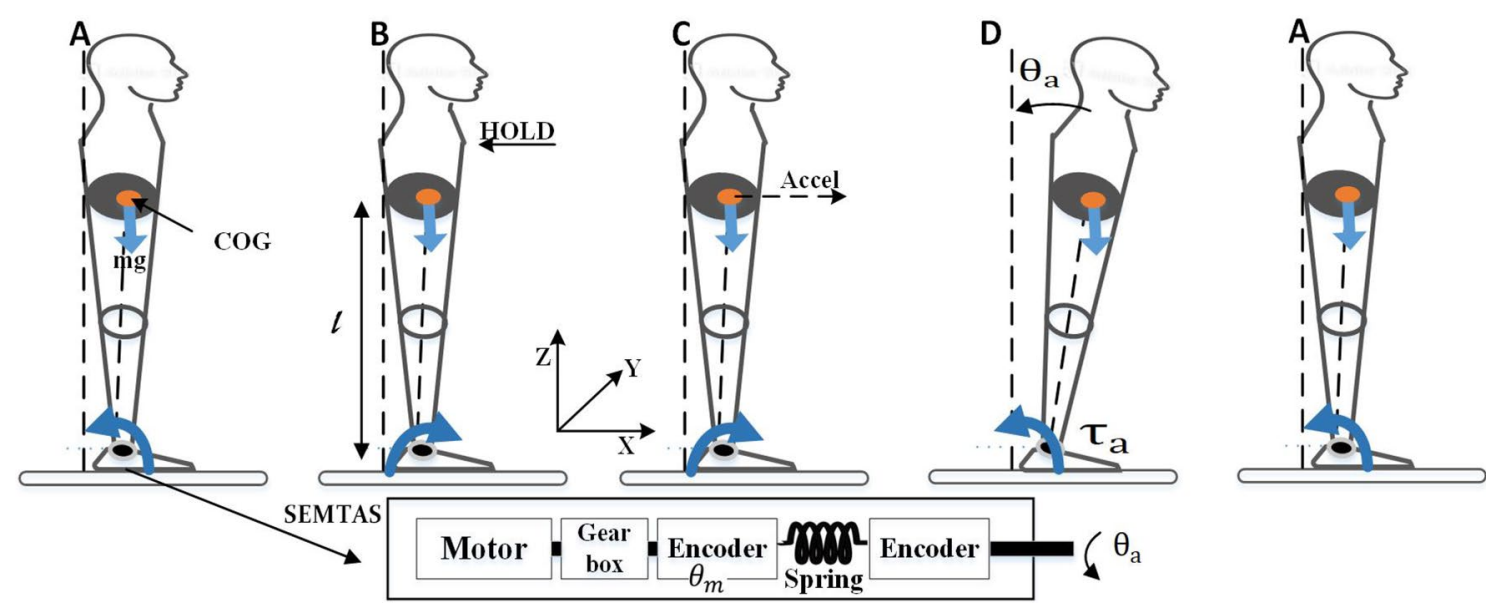

Fig. 4 Model representation of HA-SEMTAS $\left(\boldsymbol{\theta}_{\mathrm{a}}\right.$ and $\boldsymbol{\tau}_{\mathrm{a}}$ are the angle and torque of the ankle joint), quiet standing (a), hold (b), release (c) with consequence acceleration of the center of mass forward

$\theta_{a}$ is the body pendulums sway from vertical line (Fig. 4 ) and $I$ is moment of inertia, $F_{x}$ and $F_{z}$ are the components of gravity and inertia force applied to body pendulum's point mass $\mathrm{m}, x_{c}=I \sin \left(\theta_{a}\right), z_{c}=I \cos \left(\theta_{a}\right)$ are the point mass coordinates, $g$ is gravity acceleration $\left(\mathrm{g}=-9.81 \mathrm{~m} / \mathrm{s}^{2}\right), \mathrm{l}$ is the body pendulum's length. Following the zero moment point (ZMP) definition [13], in equilibrated posture the torque $\tau_{a}$ is compensated by the torque $\tau_{a r}$ from the reaction force $\left(F_{x r}, F_{z r}\right)$ are the reaction force components). Assuming that the ankle joint is on the ground level (it means $\mathrm{h}=0$ what is a common assumption), and taking into account that $F_{x r}=-F_{x}$ ${ }^{,} F_{z r}=-F_{z}$, and $\tau_{a}+\tau_{a r}=0$, Considering (1) and (2) we obtain an expression for acceleration in the ankle joint during the free fall:

$\ddot{\theta}_{a}=\frac{1}{l} m g l \sin \left(\theta_{a}\right)$

Actively decreasing this acceleration by the ankle actuator prevents the body from falling. Ankle joint the required application point of the reaction force resulting in dynamic equilibrium. The elastic element acts as actuator for the reaction force in the ankle joint, because it transfers torque by the effect of elastic displacement. On the other hand, the amount of force can also be calculated from the measurement of elastic displacement, so that SEMTAS acts as a transducer at the same time. Therefore, both sections are in the control loop. The elastic element $k_{s}$ can be selected as a constant coefficient. It can change depending on the rigidity of the system. If the spring hysteresis is not taken into consideration, the arc length and force relation according to the Hooke rule will be as follows; stiffness profiles of TFS were identified by slowly pulling and pushing the ankle joint through an attached force sensor as the (d), recoil where fall is prevented and quiet posture is regained as in condition (a) [10].

motor held the "proximal" position of the SEMTAS constant (spring stiffness: $\mathrm{k}_{\mathrm{s}}=63.655 \mathrm{Nm} / \mathrm{rad}$ ).

$\tau_{m}+k_{s}\left(\theta_{a}-\theta_{m}\right)=J_{m} \ddot{\theta}_{m}$

$\tau_{a r}=-k_{s}\left(\theta_{a}-\theta_{m}\right)$

if $\left(\ddot{\theta}_{a}=0\right)$ (speed constant) let's assume and ( $\omega=$ Natural frequency)

$\frac{\tau_{a r}}{\tau_{m}}(s)=\frac{1}{1+\frac{J_{m}}{k_{s}} s^{2}}, \quad \omega=\sqrt{\frac{k_{s}}{J_{m}}}$

$\tau_{a r}(s)=\tau_{m}(s) \frac{1}{1+\frac{J_{m}}{k_{s}} s^{2}}-\theta_{a}(s) \frac{s^{2} J_{m} k_{s}}{1+\frac{J_{m}}{k_{s}} s^{2}}$

where $\tau_{m}$ and $\tau_{a r}$ are the torque of the motor and the ankle respectively, $J_{m}$ is the inertia of the motor, $k_{s}$ is the stiffness of the torsional flat Spring, $\theta_{m}$ and $\theta_{a}$ are the angles of the motor shaft and the ankle joint respectively, $\mathrm{m}$ is body pendulum's point mass.

\subsection{Controller design}

The type of motor used in the system effect on the control performance of the HA-SEA. In this section, a PID torque controller [14] design for HA-SEMTAS will be explained in detail.

During balancing, the ankle torque has to cope with the whole body [15]. The corresponding ankle control model is depicted in Fig. 5 . 


\subsection{FeedForward model}

$\tau_{m}(s)=\left(1+\frac{J_{m}}{k_{s}} s^{2}\right) \tau_{a r}(s)+J_{m} s^{2} \theta_{a}(s)$

As $T_{\text {ideal }}$ is fixed, its second derivative will be zero.

$\tau_{m}=\tau_{\text {ideal }}+J_{m} s^{2} \theta_{a}$

Recently works have been shown that force control performance and stability can be improved with series damper components [16]. In particular, the series dampers can augment force control bandwidth (when coupled with high impedance environment) and can reduce oscillations that can arise when coupled with low impedance environment.

Also, the series dampers increase Z-width. The series elastic/damping actuator model can be written as.

$\tau_{a r}=k_{s}\left(\theta_{m}-\theta_{a}\right)+d_{s}\left(\dot{\theta}_{m}-\dot{\theta}_{a}\right)$

$J_{m} \ddot{\theta}=\tau_{m}-\tau_{a r}$

where $d_{s}$ is the equivalent series damping coefficient [17], $\tau_{a}$ is the torque exerted on the environment and the measured torque is still the spring torque $\tau_{a r}=k_{s}\left(\theta_{m}-\theta_{a}\right)$. In the Fig. 5 below is included a drawing of the proposed control scheme-it includes the feedforward terms and the PID control loop Eq. (10). According to the results we have determined the PID parameters. The block diagram of proposed The PID controller is shown in Fig. 5. As mentioned before, the PID gains are obtained by using genetic algorithm [18]. The genetic algorithm characteristics are population type $=$ double vector, generations number $=100$, scaling function $=$ rank, selection function $=$ stochastic uniform, mutation function $=$ adaptive feasible, crossover function = scattered [19], and the PID parameters are $P$ (proportional gain) $=2.73$, I (integral gain $)=3.94$, and $D$ (derivative gain $)=1.34$.

\section{Experimental setup}

In experimental setup, a torque motor with the worm gear is employed, used for rotational force and as shown in Fig. 6. The motor and load encoder sensors give information about motor angle and load angle respectively to the control unit. Figure 6 illustrates the experimental setup of the HA-SEMTAS controlled by PID controller [20] embedded in Industrial PC. Special torque motor with speed of $270 \mathrm{rpm}$ and voltage of $48 \mathrm{~V}$ and worm gear with ratio of 1:60 are used in experimental setup. Furthermore, encoders sensor (HE40-50B) and the Driver (EC-10A) are included in the system. Encoder sensors are 2048 bit. The control block circuit includes PWM Generator card (ATMEGA128 microcontroller) which handles the main task of controlling and filtering inputs and outputs of the HA-SEMTAS and an Industrial PC Controller (GoogolThech GT-800 Series Motion Controller) (Table 2).

\section{Results and discussions}

Figure 7 illustrates position signals obtained from the experimental setup (GoogolThech GT-800 Series Motion Controller), in order to see the behavior of the controller performance. The evaluation of the controller performance is performed using IAE, ISE, and ITSE. A dynamic performance comparison of the two controllers is given in Table 3. Figure 8 shows the comparison of controller response for a step input (Table 4).

Step input response of the HA-SEMTAS for PID and $\mathrm{PD}+\mathrm{ff}$ controller is shown in Fig. 7.

As shown in Fig. 9, the satisfied performance of torque tracking and external disturbance response ability are obtained during human standing position.

This reference input resembles a human ankle in a standing position.
Fig. 5 Block diagram of the control model of HA-SEMTAS

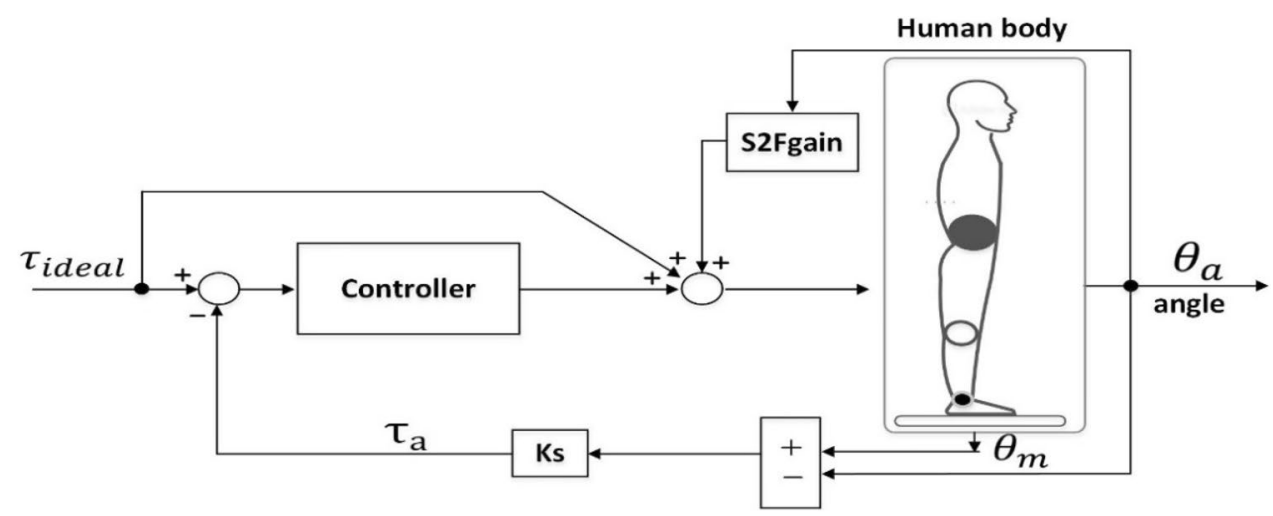

SN Applied Sciences 


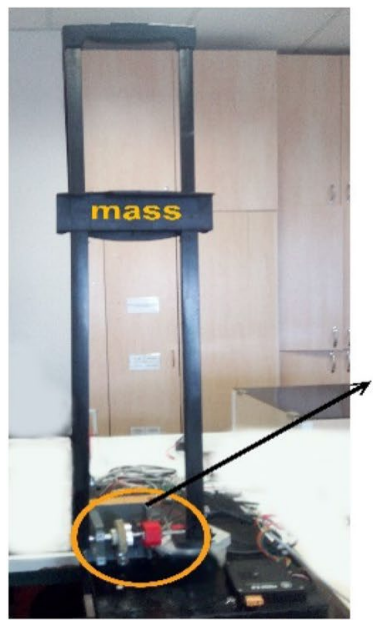

a

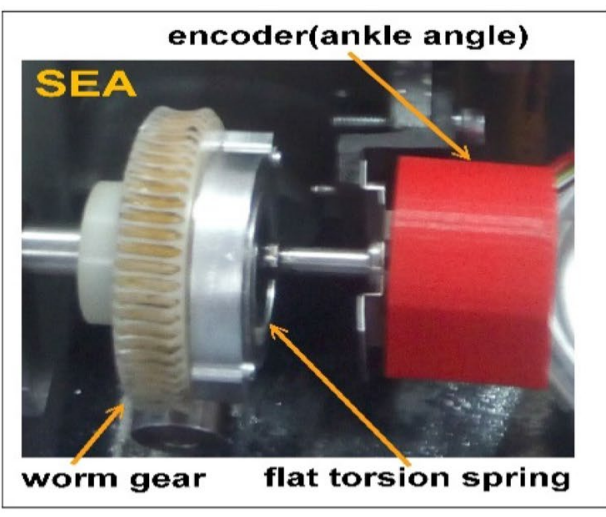

b

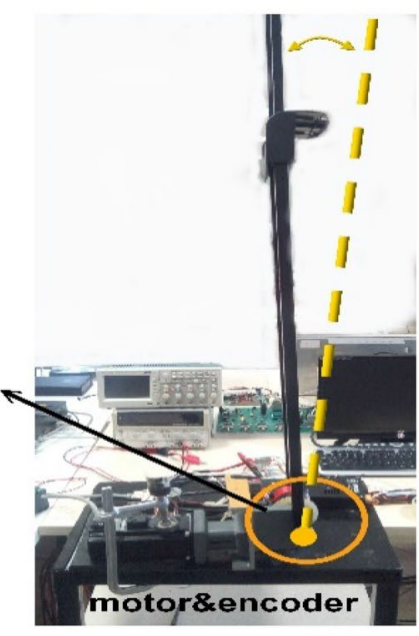

C

Fig. 6 Experimental setup: a general view, b close look of worm gear, torsion spring and encoder, $\mathbf{c}$ motor angle

Table 2 System parameters

\begin{tabular}{lll}
\hline Parameter & Symbol & Mean value \\
\hline Spring stiffness & $k_{s}$ & $63.665 \mathrm{Nm} / \mathrm{rad}$ \\
Torque constant & $k_{t}$ & $2.391 \mathrm{Nm} / \mathrm{A}$ \\
Motor inertia & $J_{m}$ & $0.00032 \mathrm{~kg} / \mathrm{m}^{2}$ \\
\hline
\end{tabular}
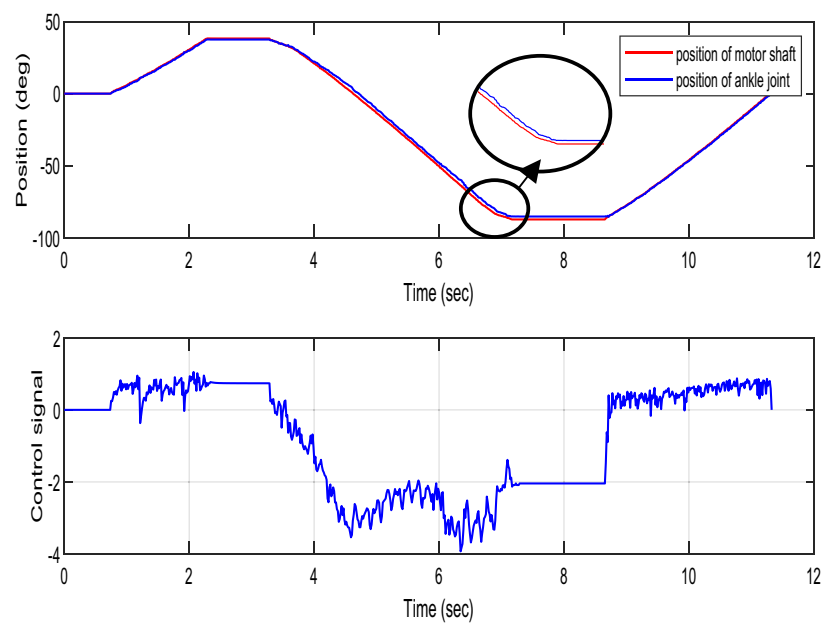

Fig. 7 Performance of PID position control of HA-SEMTAS.

Table 3 Comparison of the controllers based on different performance index

\begin{tabular}{llll}
\hline Controller & IAE & ISE & ITSE \\
\hline PID controller & 0.558 & 0.636 & 0.550 \\
PD +ff controller & 0.186 & 0.150 & 0.077 \\
\hline
\end{tabular}

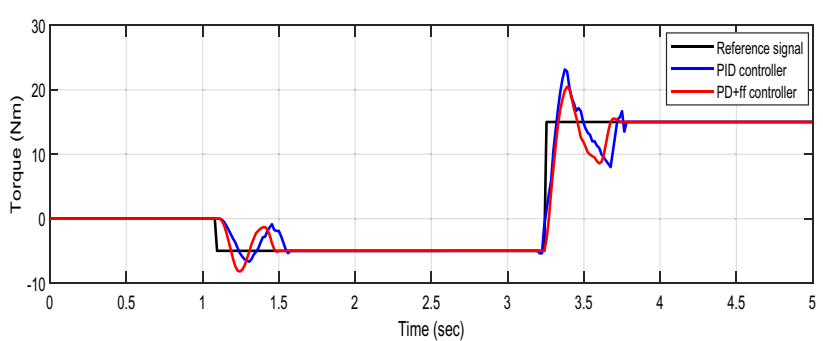

Fig. 8 Step input response of HA-SEMTAS for PID and PD + ff controller

Table 4 Comparison of performance results for PID and PD + ff

\begin{tabular}{llll}
\hline Controller & Ris time & Settling time & $\begin{array}{l}\text { Maximum } \\
\text { overshoot } \\
\%\end{array}$ \\
\hline PID controller & 0.065 & 0.525 & 40.707 \\
PD +ff controller & 0.077 & 0.495 & 27.364 \\
\hline
\end{tabular}

\section{Conclusions and future work}

In this paper, a torsional flat spring (TFS) is proposed to provide high compliance and deformation values for Human Ankle Series Elastic muscle-tendon Actuator System (HA-SEMTAS). Also, a dynamic model and control of the HA-SEMTAS was developed under internal and external disturbances. Using this model, two different controllers (PID and PD + ff) were applied to HA-SEMTAS. The results obtained indicate that the $\mathrm{PD}+\mathrm{ff}$ controller performs well for high frequency disturbances. Reference 

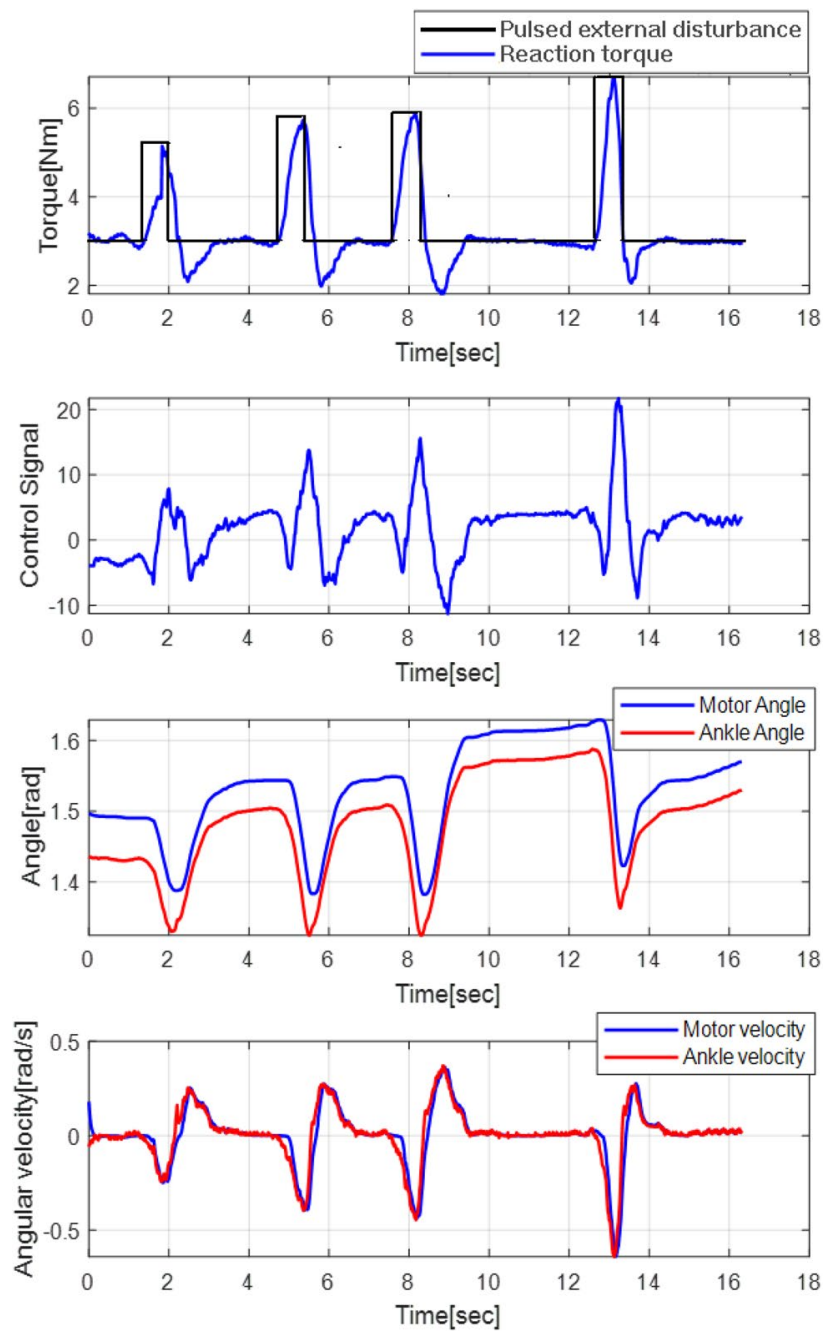

Fig. 9 Torque disturbance: pulsed external disturbance and ankle reaction torque $\left(\tau_{a r}\right)$, control signal, position of the motor and ankle joint and velocity of the motor and ankle joint

trajectories are followed with reasonable time delay. The real-time torque controller for HA-SEMTAS was embedded in the Industrial PC Controller (Motion Controller Series model: GoogolThech GT-800). The PID and PD + ff parameters in the controller with the inverse dynamic model were tuned by using the genetic algorithm. The $\mathrm{PD}+\mathrm{ff}$ controller with SEA enhances the stability and control performance of the HA-SEMTAS. In future work, more in-depth investigations into the behavior of the system for slow frequency disturbances will be included which will be compared with human responses. Also, a real human movement will test in a moving Stewart platform.

Acknowledgements I would like to thank The Presidency of Turks Abroad and Related Communities (YTB) for funding this doctoral studies research.

\section{Compliance with ethical standards}

Conflict of interest The authors declare that they have no conflict of interest.

\section{References}

1. Kim S, Horak FB, Carlson-Kuhta P, Park S (2009) Postural feedback scaling deficits in Parkinson's disease. J Neurophysiol 102(5):2910-2920

2. Popovic M, Hofmann A, Herr H (2004) Zero spin angular momentum control: definition and applicability. In: 4th IEEE/RAS international conference on humanoid robots, 2004, vol 1, pp 478493). IEEE

3. Mergner T (2010) A neurological view on reactive human stance control. Annu Rev Control 34(2):177-198

4. Zebenay M, Lippi V, Mergener T (2015) Human-like humanoid robot posture control. In: 2015 12th International conference on informatics in control, automation and robotics (ICINCO), vol 2, pp 304-309. IEEE

5. Cho BK, Kim JY (2018) Dynamic posture stabilization of a biped robot SUBO-1 on slope-changing grounds. Int J Precis Eng Manuf 19(7):1003-1009

6. Ott C, Roa MA, Hirzinger G (2011) Posture and balance control for biped robots based on contact force optimization. In: 2011 11 th IEEE-RAS international conference on humanoid robots, pp 26-33. IEEE

7. Stephens BJ, Atkeson CG (2010) Dynamic balance force control for compliant humanoid robots. In: 2010 IEEE/RSJ international conference on intelligent robots and systems, pp 1248-1255. IEEE

8. Endo K, Herr H (2009). A model of muscle-tendon function in human walking. In: 2009 IEEE international conference on robotics and automation, pp 1909-1915. IEEE

9. Chen T, Casas R, Lum PS (2019) An elbow exoskeleton for upper limb rehabilitation with series elastic actuator and cable-driven differential. IEEE Trans Robot 35:1464-1474

10. Chavez-Romero R, Cardenas A, Maya M, Vernaza KM, Piovesan D (2014) Experimental validation of vision-based system for the characterization of human standing. In: Proceedings of the Latin American congress of automatic control (IFACCLCA)

11. Fotuhi MJ, Bingul $Z$ (2018) Position and trajectory fuzzy control of a laboratory 2 DOF double dual twin rotor aerodynamical system. In: 2018 IEEE 27th international symposium on industrial electronics (ISIE), pp 277-282. IEEE

12. Zielinska T, Gao Z, Zurawska M, Zheng Q, Mergner T, Lippi V (2017) Postural balance using a disturbance rejection method. In: 201711 th International workshop on robot motion and control (RoMoCo), pp 23-28. IEEE

13. Sharma MK, Ordonez R, Sutton G (2018). Modelling and applications of a variable spring series elastic actuator. In: 2018 27th IEEE international symposium on robot and human interactive communication (RO-MAN), pp 66-71. IEEE

14. Knabe C, Lee B, Orekhov V, Hong D (2014) Design of a compact, lightweight, electromechanical linear series elastic actuator. In: ASME 2014 international design engineering technical conferences and computers and information in engineering conference, pp V05BT08A014-V05BT08A014. American Society of Mechanical Engineers

15. Yu H, Huang S, Chen G, Pan Y, Guo Z (2015) Human-robot interaction control of rehabilitation robots with series elastic actuators. IEEE Trans Rob 31(5):1089-1100 
16. Fotuhi MJ, Hazem ZB, Bingül Z (2019). Modelling and torque control of an non-linear friction inverted pendulum driven with a rotary series elastic actuator. In: 2019 3rd International Symposium on Multidisciplinary Studies and Innovative Technologies (ISMSIT). IEEE, pp 1-6

17. Oh S, Kong K (2016) High-precision robust force control of a series elastic actuator. IEEE/ASME Trans Mechatron 22(1):71-80

18. Gani MM, Islam MS, Ullah MA (2019) Optimal PID tuning for controlling the temperature of electric furnace by genetic algorithm. SN Appl Sci 1(8):880
19. Cummings JP, Ruiken D, Wilkinson EL, Lanighan MW, Grupen RA, Sup FC (2016) A compact, modular series elastic actuator. J Mech Robot 8(4):041016

20. Kumar S, Jayaswal K, Kothari DP (2019) Investigation of feasible controller for position control of flexible joint manipulator using multiple control techniques. SN Appl Sci 1(12):1634

Publisher's Note Springer Nature remains neutral with regard to jurisdictional claims in published maps and institutional affiliations. 